\title{
Exact Solution for Thermal Stagnation-Point Flow with Surface Curvature and External Vorticity Effects
}

\author{
Ronald Ming Cho So ${ }^{1}$, Elizabeth Wing Sze Kam ${ }^{2 *}$ \\ ${ }^{1}$ Mechanical Engineering Department, the Hong Kong Polytechnic University, Hong Kong, China \\ ${ }^{2}$ Department of Mathematical Sciences, Rensselaer Polytechnic Institute, Troy, NY, USA \\ Email: mmmcso@polyu.edu.hk, ^kamwingsze@gmail.com
}

How to cite this paper: So, R.M.C. and Kam, E.W.S. (2017) Exact Solution for Thermal Stagnation-Point Flow with Surface Curvature and External Vorticity Effects. Journal of Applied Mathematics and Physics, 5, 966-989.

https://doi.org/10.4236/jamp.2017.54085

Received: February 19, 2017

Accepted: April 27, 2017

Published: April 30, 2017

Copyright $\odot 2017$ by authors and Scientific Research Publishing Inc. This work is licensed under the Creative Commons Attribution International License (CC BY 4.0).

http://creativecommons.org/licenses/by/4.0/

\begin{abstract}
Exact solution of the steady Navier-Stokes equations has been obtained for the thermal stagnation-point flow at the leading edge of a turbine blade under the assumptions of constant nose radius and external vorticity, and fluid properties independent of temperature. The solutions reveal that curvature affects local heat transfer and skin friction while external vorticity does not. The effect of external vorticity is to shift the zero skin friction point away from the stagnation point. This solution is valid for all Reynolds number, external vorticity, and nose radius. In the limit of nose radius going to infinity and external vorticity, going to zero, the exact solution for two-dimensional plane stagnation-point flow is recovered identically. In addition, it can be shown that the velocity field around the stagnation point of a rotating curved surface is the same as that around the stagnation point of a stationary curved surface with an external vorticity which equals to twice of the rotational speed. This realization renders the present solution equally valid for thermal stagnationpoint flow at the leading edge of centrifugal impeller blades.
\end{abstract}

\section{Keywords}

Navier-Stokes Equations, Exact Solutions, Thermal Stagnation-Point Flow, Displacement Effect

\section{Introduction}

Frequently, in the past, two-dimensional calculation methods were used to determine the gas-side heat transfer coefficient on turbine blades [1]. All these methods, whether based on the integral or differential equations, were derived on the assumption that the flow is steady and the static pressure variation across 
the boundary layer has very little effect on the flow, and hence can be neglected [2]. Measurements of two-dimensional turbulent boundary layers along plane surfaces lend support to this assumption [3]. However, when these methods were used to calculate flows along curved surfaces, they were found to be inadequate comparing with measured data [2]. The reason is that existing two-dimensional method neglects the effect of curvature on the mean flow streamlines. This neglect is justifiable in laminar flows [4] [5] if $k \delta \ll 1$. On the other hand, the neglect of the effect of streamline curvature is not justifiable in the case of turbulent flows [6] even when $k \delta \ll 1$.

Striking effects of curvature have been observed in turbulent boundary-layer flow over convex and concave surfaces [7] [8]. Boundary layer measurements showed that large convex curvature in the mean flow streamlines leads to vanishing shear stress in regions where the mean velocity gradient is still substantial [7], while large concave curvature promotes the formation of Taylor-Gortler type instabilities [8]. As a result, various attempts were made by different investigators to account for the effect of streamline curvature in two-dimensional turbulent shear flows [9]-[14]. Attempt to use these techniques to calculate flow around compressor blades was first made in [13], and good correlations were obtained between measured and calculated results. Furthermore, these techniques were extended to model heat transfer on curved surfaces alone [14] and curved surfaces with swirl [15]. Again, good correlations were obtained between measured and calculated heat transfer results. In view of this, the same technique has also been used to predict the flow and heat transfer around axial flow turbine blades where Coriolis effects are absent [15].

Generally, the solution of the two-dimensional plane stagnation-point flow [16] [17] [18] is used to provide initial conditions for heat transfer calculations along turbine blades. This assumption is valid as long as the surface curvature is considered to have negligible effect on the boundary-layer flow around the blades. With the introduction of improved techniques mentioned above [9]-[15], it is evident that, to be consistent with the improved techniques, the effect of surface curvature on the stagnation-point flow cannot be neglected.

The boundary layer thickness in a stagnation-point flow is given by $\delta=2.4 \sqrt{v / a}$; therefore, it can be seen that the neglect of curvature effect is valid only if $\sqrt{v / a}$ is much less than the radius of curvature of the surface at the stagnation point [18]. In the leading edge of a turbine blade, the nose radius is very small and the above condition is not necessary true. As a result, surface curvature may have a significant effect on the flow and heat transfer downstream of the forward stagnation of a turbine blade. In addition to being affected by surface curvature $(k)$, the flow and heat transfer at the leading edge are also influenced by free stream vorticity $(\Omega)$ in the flow approaching the turbine blades. Therefore, to obtain the correct initial conditions for subsequent heat transfer calculations around the blades using any one of the previously mentioned techniques [9]-[15], the effect of $k$ and $\Omega$ on the flow and heat transfer 
at the leading edge have to be considered and analyzed.

Effect of surface curvature on stagnation-point flow was first examined in [4] [5]. Assuming that $R e \gg 1$ and $k \delta \ll 1$, the analysis in [4] [5] proceeded to expand the stream function in terms of $1 / R e$. The problem was then solved using the technique of matched asymptotic expansions and the results gave the second-order effect on heat transfer coefficient and skin friction due to surface curvature. Further, second-order effect due to flow displacement and freestream $\Omega$ was also evaluated [4] [5]. Subsequently, other investigators [19] [20] [21] have also carried out analysis on the same problem invoking the same assumptions, but using different methods to solve the problem. The results obtained by these investigators were no different than those presented earlier [4] [5]. In some cases [19] [20], the results were shown to be less accurate because of the assumptions the investigators had to invoke to simplify the problem.

The effect of constant external $\Omega$ on two-dimensional plane stagnationpoint flow was also attempted and an exact solution to the governing equations was obtained [22]. Unlike the perturbation solution mentioned previously [4] [5], the exact solution, without accounting for the effect of flow displacement, is valid for all Re [22]. Further, it was found that the effect of external $\Omega$ on the flow was to shift the zero skin friction point away from the stagnation point; however, no attempt had been made to analyze the heat transfer problem [22]. On the other hand, a complete second-order analysis of the problem was carried out and it showed that if displacement effect were also included [23], the shift of the zero skin friction point from the stagnation point is greater than that predicted in [22]. A thorough discussion of most second-order effects has been presented in [23] where the flows considered are not limited to stagnation-point flows.

In spite of the fairly complete second-order treatment of stagnation-point flow on curved surfaces [4] [5] [23], the analysis is rather limiting because of the assumptions of $\operatorname{Re} \gg 1$ and $k \delta \ll 1$. These assumptions could lead to two sources of error in the prediction of gas-side heat transfer coefficients on turbine blades. The obvious source is the inaccurate estimate of the heat transfer coefficient at the leading edge, which may or may not be too critical depending on the value of $R e$ and $k \delta$ at the nose. A second source of error is associated with the incorrect estimate of initial conditions for subsequent convective heat transfer calculations on the rest of the blade. The effect of this error on the calculated heat transfer coefficient is difficult to estimate, and it could be more severe as $R e$ decreases and $k \delta$ increases at the leading edge. Consequently, there is a need for a more exact theory that can correctly account for the effect of surface curvature and external $\Omega$ on thermal stagnation-point flows at the leading edge of turbine blades.

The objective of the present paper, therefore, is to investigate the effect of surface curvature and constant external $\Omega$ on heat transfer at the leading edge of axial flow turbine blades. To simplify the problem, the flow near the leading edge of the blade is approximated by the flow around a two-dimensional cylinder of 
the same radius as the nose. Also, the flow is assumed to be steady and laminar, and the fluid properties are assumed constant over the temperature range of interest. As the first attempt, the assumption of laminar flow at the stagnation point and its immediate vicinity is reasonable. It is shown that exact solutions to the governing equations could be obtained and that these solutions would reduce to the exact solution with constant $\Omega$ [22] as $k \rightarrow 0$, and to the classical solutions [15] [16] [17] [18] in the limit of zero surface curvature and zero external $\Omega$. Thus formulated, the solutions are valid for all values of $R e, k \delta$ and $\Omega$. Once this solution is obtained, it is shown that the formulation can be extended to analyze the thermal stagnation-point flow at the leading edge of centrifugal impeller blades.

\section{The Governing Equations}

The governing equations describing the steady incompressible flow and temperature fields near the forward stagnation point of a two-dimensional cylinder of constant radius $R$ can be written with respect to a co-ordinate system attached to the cylinder as shown in Figure 1. In component form, the full set of steady Navier-Stokes equations [24] is reduced to:

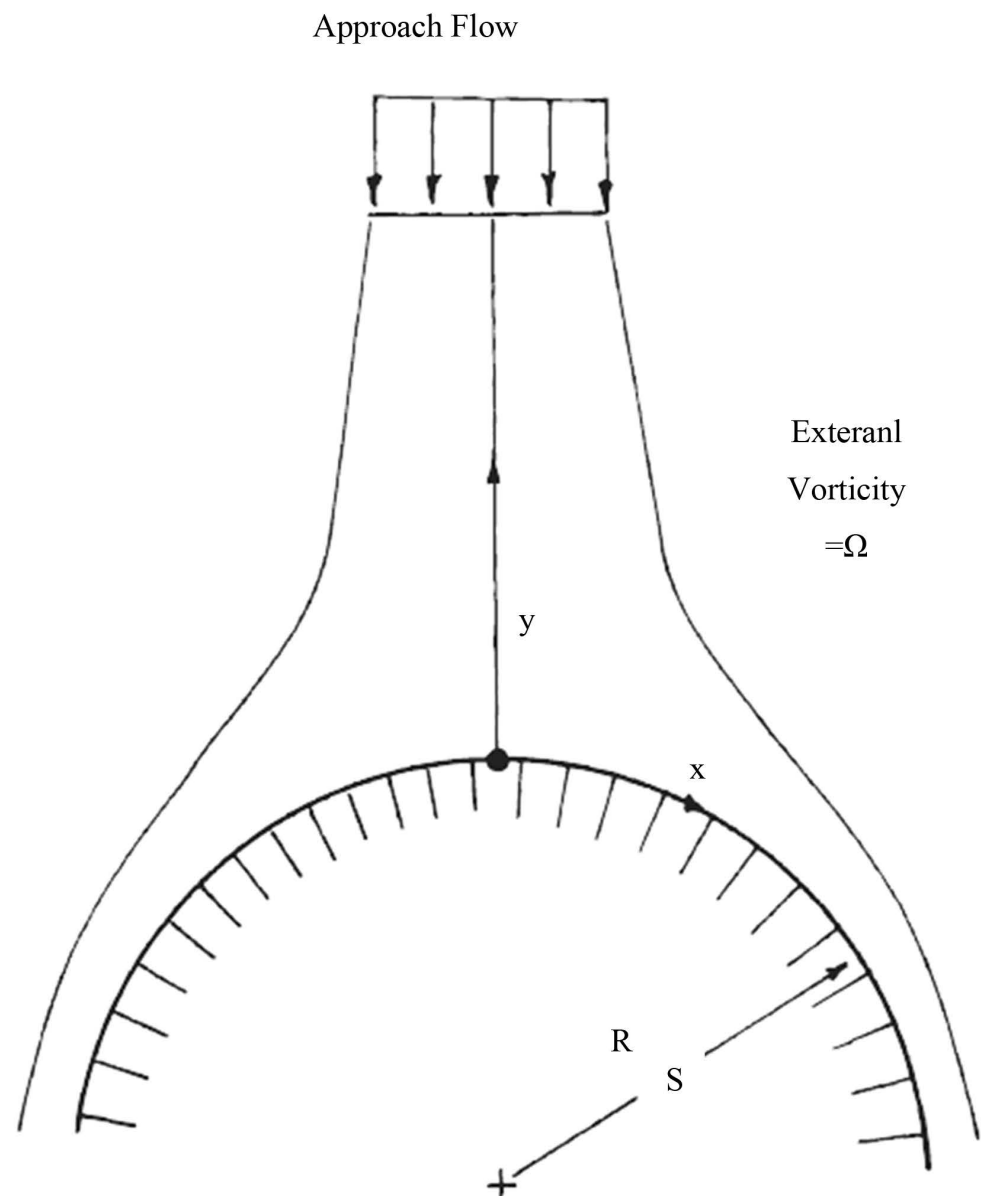

Figure 1. Curvilinear co-ordinate system for a stationary surface. 


$$
\begin{gathered}
u_{x}+(h v)_{y}=0, \\
u u_{x}+v(h u)_{y}=-\rho^{-1} p_{x}+v\left[h^{-1} u_{x x}+h u_{y y}+k u_{y}-k^{2} h^{-1} u+2 k h^{-1} v_{x}\right], \\
u v_{x}+h v v_{y}-k u^{2}=-\rho^{-1} h p_{y}+v\left[h^{-1} v_{x x}+h v_{y y}+k v_{y}-k^{2} h^{-1} v-2 k h^{-1} u_{x}\right], \\
u T_{x}+h v T_{y}=\alpha\left[h^{-1} T_{x x}+h T_{y y}+k T_{y}\right],
\end{gathered}
$$

where subscripts denote partial differentiation, $k$ is positive for convex and negative for concave curvature, and viscous dissipation of heat has been assumed negligible. The boundary conditions are no slip at the wall, uniform flow and temperature far upstream of the stagnation point, and inviscid flow with a constant $\Omega$ outside a shear layer close to the wall.

Assuming constant fluid properties allow the velocity field equations to be decoupled from the temperature field equation. A complete solution to the aerodynamic problem, i.e. Equations (1)-(3), can be obtained by first finding a solution to the outer inviscid flow and then proceeding to determine the flow in the viscous region near the wall. Once the velocity field near the stagnation point is known heat transfer to or from the cylinder surface can be obtained by solving Equation (4) with the appropriate temperature boundary conditions.

\section{The Inviscid Solution}

Since the flow in the outer region is inviscid, the stream function, $\Psi(x, y)$, satisfies the Poisson equation, which can be written as

$$
h^{-1}\left(h^{-1} \Psi_{x}\right)_{x}+h^{-1}\left(h \Psi_{y}\right)_{y}=-\Omega,
$$

where the stream function is defined by

$$
\begin{gathered}
u=\Psi_{y}, \\
h v=-\Psi_{x} .
\end{gathered}
$$

The boundary conditions are

$$
\begin{gathered}
\Psi=0 \text { on the body, } \\
\Psi(x, y) \sim \Psi_{\infty} \text { far upstream. }
\end{gathered}
$$

This problem can be considered properly set for an elliptic differential equation, and a solution for $\Psi$ can be sought in the form

$$
\Psi(x, y)=P(x) Q(y)+\Omega S(y) .
$$

Substituting (10) into (5) gives

$$
\begin{gathered}
-\frac{P^{\prime \prime}}{P}=\frac{h^{2} Q^{\prime \prime}}{Q}+\frac{k h Q^{\prime}}{Q}=n^{2}, \\
h^{2} S^{\prime \prime}+k h S^{\prime}=-h^{2},
\end{gathered}
$$

where the primes denote ordinary differentiation with respect to $x$ for $P$, and to $y$ for $Q$ and $S$. If the solution for $\Psi(x, y)$ is to approach the classical two-dimensional plane stagnation-point flow solution when $k \rightarrow 0$ and $\Omega \rightarrow 0$, then 
it can be shown that the only meaningful solution for (11) is given by $n=0$. With $n=0$, solutions for $P(x)$ and $Q(y)$ are

$$
\begin{aligned}
& P(x)=a_{1} x, \\
& Q(y)=\frac{a_{2} \ln h}{k},
\end{aligned}
$$

where $a_{1}$ and $a_{2}$ are arbitrary constants. Since the homogeneous solution of (12) is identical to that obtained for $Q$ and $\Omega$ is constant, it can be easily shown that only the particular integral of Equation (12) is of interest. A particular solution of (12) is given by

$$
S(y)=-\frac{y(1+h)}{4 k}+\frac{\ln h}{2 k^{2}} .
$$

As a result, the solution for $\Psi$ with boundary conditions (8) and (9) can be written as:

$$
\Psi(x, y)=\frac{a x}{k} \ln h-\frac{\Omega y(1+h)}{4 k}+\frac{\Omega}{2 k^{2}} \ln h,
$$

where $a$ is an arbitrary constant. It should be pointed out that in the limit of $k \rightarrow 0$, Equation (16) reduces to the solution given in [22], or

$$
\lim _{k \rightarrow 0} \Psi=\operatorname{axy}-\frac{1}{2} \Omega y^{2} .
$$

Therefore, when $\Omega=0$, Equation (17) reduces to the classical potential solution for two-dimensional plane stagnation-point flow.

The velocity field is given by

$$
\begin{gathered}
u=\frac{a x}{h}-\frac{\Omega y(1+h)}{2 h}, \\
v=-\frac{a \ln h}{k h},
\end{gathered}
$$

and the pressure field is obtained by integrating the Euler equations which are the inviscid counterparts of (2) and (3). With the help of (18) and (19), the pressure field can be written as:

$p_{0}-p=\frac{1}{2} \rho\left[\frac{a^{2} x^{2}}{h^{2}}+\frac{a \Omega x}{k} \frac{2 h^{2} \ln h-h^{2}+1}{h^{2}}+\frac{\Omega^{2}}{4 k^{2}} \frac{h^{4}-4 h^{2} \ln h-1}{h^{2}}+\frac{a^{2}(\ln h)^{2}}{k^{2} h^{2}}\right]$,

where $p$ is the pressure and $p_{0}$ is the pressure at the stagnation point. It should be noted that even when $\Omega \rightarrow 0$ the velocity u downstream of the stagnation point is not uniform as could be seen from Equation (18). This is necessary because curvature gives rise to a "centrifugal force" that is balanced by a normal pressure gradient.

\section{Viscous Flow and Heat Transfer}

Equations (18)-(20) represent a complete solution to the inviscid problem. Once the inviscid solution is known, the next task is to focus on the viscous flow and 
heat transfer near the surface. Since the inviscid solution does not satisfy the no-slip condition at the wall, a viscous solution that is valid at and near the surface will be sought. This solution has to approach (18) and (19) for large $y$, and in this limit, the vorticity also approaches $\Omega$ in the external flow. In order not to impose any conditions on $R e, k \delta$, and $\Omega$, an exact solution to the governing Navier-Stokes Equations (1)-(3) is sought in the viscous layer. The pressure terms in the momentum equations are eliminated by cross-differentiating (2) and (3), and the resulting vorticity equation is given by

$$
\begin{aligned}
& h^{2} u u_{x y}+h^{3} v u_{y y}+k h^{2} u v_{y}+k h^{2} v u_{y}-h u v_{x x}-h^{2} v v_{x y}+h k v v_{x}+2 k h u u_{x} \\
& =v\left[h u_{x x y}+k u_{x x}+h^{3} u_{y y y}+2 k h^{2} u_{y y}-k^{2} h u_{y}+k^{3} u+k h v_{x y}-k^{2} v_{x}-v_{x x x}-h^{2} v_{x y y}\right],
\end{aligned}
$$

where use has been made of Equation (1). Stagnation-point flow near the surface is, therefore, given by the solution of (21) subject to the boundary conditions

$$
\left.\begin{array}{c}
u(x, y)=v(x, 0)=0, \\
u(x, y) \rightarrow \frac{a x}{h}-\frac{\Omega y(1+h)}{2 h} \\
h^{-1} v_{x}-h^{-1}(h u)_{y} \rightarrow \Omega
\end{array}\right\} \text { for large } y .
$$

Once the velocity field is known, the stagnation-point heat transfer problem can be analyzed by solving Equation (4) subject to the conditions of an isothermal body and a uniform temperature in the external flow.

As in the case of plane flows with or without the effect of $\Omega$ [15] [16] [17] [18] [22], a similar solution to Equation (21) is sought such that the velocity and temperature fields can be written as:

$$
\begin{gathered}
u=a x F^{\prime}(\eta)-\Omega \sqrt{\frac{v}{a}} \frac{G(\eta)}{h}, \\
v=-\sqrt{a v} \frac{F(\eta)}{h}, \\
T=T_{w}+\left(T_{\infty}-T_{w}\right) \Theta(\eta),
\end{gathered}
$$

where $\eta=(\sqrt{a / v}) y \quad$ is the similarity variable, $h=1+k y=1+K \eta \quad$ and $K=(\sqrt{v / a}) k$. Substituting Equations (23)-(25) into (21) and (4), the following equations for $F(\eta), G(\eta)$, and $\Theta(\eta)$ are obtained,

$$
\begin{gathered}
F^{\prime \prime \prime}+\frac{F+2 K}{h} F^{\prime \prime \prime}+\frac{K(F-K)-h F^{\prime}}{h^{2}} F^{\prime \prime}-\frac{K^{2}(F-K)+K h F^{\prime}}{h^{3}} F^{\prime}=0, \\
G^{\prime \prime \prime}+\frac{F-K}{h} G^{\prime \prime}+\frac{K(F-K)}{h^{2}} G^{\prime}-\frac{h F^{\prime \prime}+K F^{\prime}}{h^{2}} G=0, \\
\Theta^{\prime \prime}+\frac{F P r+K}{h} \Theta^{\prime}=0,
\end{gathered}
$$

where the primes denote ordinary differentiation with respect to $\eta$. The conditions that $T(x, 0)=T_{w}, T(x$, large $y)=T_{\infty}$ and (22) give the necessary boundary conditions for (26)-(28).

It is not convenient to choose $G(\eta)$ at large $\eta$ as one of the outer boun- 
dary conditions for $G$ because its value at large $\eta$ is dependent on displacement. As a result, the outer boundary conditions for $G$ are defined by the functions $G^{\prime}(\eta)$ and $G^{\prime \prime}(\eta)$ at large $\eta$. In terms of $\eta$, the boundary conditions for $F$, $G$, and $\Theta$ can then be written as

$$
\begin{aligned}
& \text { at } \eta=0: F(0)=0 \text {, } \\
& F^{\prime}(0)=0 \text {, } \\
& G(0)=0 \text {, } \\
& \Theta(0)=0 \text {, } \\
& F^{\prime \prime}(\eta)=-K h^{-2} \text {, } \\
& G^{\prime}(\eta)=h, \\
& G^{\prime \prime}(\eta)=K, \\
& \Theta(\eta)=1 \text {. }
\end{aligned}
$$

The viscous flow and heat transfer problem at the stagnation point is reduced to solving three ordinary differential equations governing $F(\eta), G(\eta)$ and $\Theta(\eta)$, and subject to boundary conditions (29) and (30). Thus formulated, solutions of these equations will represent exact solutions to the thermal stagnation-point problem at the leading edge of axial flow turbine blades.

\section{Numerical Integration of the Governing Equations}

The ordinary differential equations governing $F, G$, and $\Theta$ are highly nonlinear and analytic solutions for (26)-(28) are not easily obtainable and are not presently available. However, numerical technique could be used to simulate the solutions for (26)-(28). Since numerical solutions of ordinary differential equations is far better developed than those of highly nonlinear partial differential equations, such as the Navier-Stokes equations, and a lot more accurate, therefore, numerical solutions of (26)-(28) are both accurate and reliable.

To further simplify the governing equations for numerical analysis, a transformation first put forward in [20] is adopted. If the new similarity variable is denoted by

$$
\zeta=\frac{\ln (1+K \eta)}{K},
$$

and the new functions for $F, G$, and $\Theta$ are written as

$$
\begin{gathered}
f(\zeta)=F(\eta), \\
g(\zeta)=G(\eta), \\
\theta(\zeta)=\Theta(\eta),
\end{gathered}
$$

then it can be easily shown that in terms of $f, g$, and $\theta$, (26)-(28) become

$$
f^{\prime \prime \prime \prime}+(f-4 K) f^{\prime \prime \prime}+\left(4 K^{2}-2 K f-f^{\prime}\right) f^{\prime \prime}=0
$$




$$
\begin{gathered}
g^{\prime \prime \prime}+(f-4 K) g^{\prime \prime}-2 K(f-2 K) g^{\prime}-f^{\prime \prime} g=0, \\
\theta^{\prime \prime}+f \operatorname{Pr} \theta^{\prime}=0 .
\end{gathered}
$$

The boundary conditions can be obtained from (29) and (30) with the help of (31)-(34). The transformed boundary conditions are given by

$$
\begin{gathered}
\text { at } \zeta=0: f(0)=0, \\
f^{\prime}(0)=0, \\
g(0)=0, \\
\theta(0)=0, \\
\text { at large } \zeta: f^{\prime}(\zeta)=1, \\
f^{\prime \prime}(\zeta)=0, \\
g^{\prime}(\zeta)=\exp (2 K \zeta), \\
g^{\prime \prime}(\zeta)=2 K \exp (2 K \zeta), \\
\theta(\zeta)=1,
\end{gathered}
$$

where the primes now denote differentiation with respect to $\zeta$. Equations (35)-(37) are much simplified compared to (26)-(28). However, they are still highly nonlinear and two additional initial conditions each, namely, $f^{\prime \prime \prime}(0)$, $f^{\prime \prime}(0)$ and $g^{\prime \prime}(0), g^{\prime}(0)$ are required for the numerical integration of (35) and (36). Fortunately, a first integral of (35) and (36) can be obtained, whereas such is not possible for (26) and (27). These integrals can be used to estimate one of the required initial conditions for the integrations of (35) and (36).

Integrating (35) and (36) once from 0 to $\zeta$ and making use of (38a-38d) gives

$$
\begin{gathered}
f^{\prime \prime \prime}+f f^{\prime \prime}-f^{\prime 2}-K\left[4 f^{\prime \prime}-4 K f^{\prime}+2 \int_{0}^{\varsigma} f f^{\prime \prime} \mathrm{d} \zeta\right]=f^{\prime \prime \prime}(0)-4 K f^{\prime \prime}(0)=C, \\
g^{\prime \prime}+f g^{\prime}-f^{\prime} g-K\left[4 g^{\prime}+2(f-2 K) g-2 \int_{0}^{\varsigma} g f^{\prime} \mathrm{d} \zeta\right]=g^{\prime \prime}(0)-4 K g^{\prime}(0)=C_{1} .
\end{gathered}
$$

The constants $C$ and $C_{1}$ can be determined by evaluating (40) and (41) at large $\zeta$ and making use of (39a-39e) and the following conditions.

Since the effect of displacement have not been included in the present formulation for $f(\zeta)$, it follows from (39a) that

$$
f(\zeta)=\zeta-\delta^{*} \text { at large } \zeta,
$$

where $\delta^{*}$ is the displacement thickness for the $K \neq 0$ but $\Omega=0$ case. Also, the condition of constant in the external flow gives

$$
f^{\prime \prime \prime}(\zeta)=0 \text { at large } \zeta \text {. }
$$

On the other hand, the displacement effect on $g(\zeta)$ can be easily analyzed and it will be carried out in the following analysis.

From $(39$ c) it can be shown that

$$
g(\zeta)=\frac{1}{2 K}\left[\exp (2 K \zeta)-\exp \left(2 K \delta^{*}\right)\right] \text { at large } \zeta
$$


if displacement effect on $\mathrm{g}(\zeta)$ are neglected, and

$$
g(\zeta)=\frac{1}{2 K}[\exp (2 K \zeta)-1] \text { at large } \zeta,
$$

if displacement effect on $g(\zeta)$ are included. In the absence of curvature, (43a) reduces to the result given in [22] where displacement effect is not included, and (43b) reduces to the result presented in [4] [5] where displacement effect is accounted for. Therefore, two sets of solutions for $g(\zeta)$ will be obtained depending on whether $g(\zeta)$ is taken to satisfy (43a) or (43b) at large $\zeta$ in the evaluation of $C_{l}$.

With the conditions for $f$ and $g$ defined at large $\zeta$, it can be easily shown that $C$ and $C_{l}$ are given by

$$
\begin{aligned}
C= & f^{\prime \prime \prime}(0)-4 K f^{\prime \prime}(0)=-1-2 K \int_{0}^{\zeta} f f^{\prime \prime} \mathrm{d} \zeta+4 K^{2}, \\
C_{1}= & g^{\prime \prime}(0)-4 K g^{\prime}(0) \\
= & \frac{1}{2 K}\left[\exp \left(2 K \delta^{*}\right)-1\right]-\left(\delta^{*}-2 K\right) \exp \left(2 K \delta^{*}\right) \\
& +2 K \int_{0}^{\zeta}\left[g f^{\prime}-\frac{1}{2 K}\left\{\exp (2 K \zeta)-\exp \left(2 K \delta^{*}\right)\right\} \mathrm{d} \zeta,\right.
\end{aligned}
$$

if displacement effect on $g$ is neglected, or

$$
C_{1}=-\delta^{*}-2 K\left\{1-\int_{0}^{\zeta}\left[g f^{\prime}-\frac{1}{2 K}\left\{\exp (2 K \zeta)-\exp \left(2 K \delta^{*}\right)\right\}\right] \mathrm{d} \zeta\right\},
$$

if displacement effect on $g$ is included. It should be pointed out that in the limit of $K \rightarrow 0$, as expected, $(45 \mathrm{a}, \mathrm{b})$ reduce to $C_{1}=0$ and $C_{1}=-\delta_{0}^{*}$, respectively.

In the limit of $K \rightarrow 0,(40)$ and (41) together with (44) and (45a) reduce to the equations analyzed in [22]. According to [4], the solution given in [22] can only account for the kinematic effect of external $\Omega$. If the dynamic effect were to be analyzed, then the displacement effect on $g$ cannot be neglected. In other words, $C_{1}$ has to be evaluated from (45b). The second-order equation for vorticity effect analyzed in [4] [5] is inhomogeneous. However, it was shown in [4] [5] that the inhomogeneous part of the second-order equation is given by $-\delta_{0}^{*}$. This is different from the present analysis. According to (45b), the result $C_{1}=-\delta_{0}^{*}$ is true only when $K=0$; in other words, only for two-dimensional plane stagnation-point flow. Therefore, the approximate treatment [4] [5] can only be interpreted as accounting for the displacement effect resulting from a two-dimensional plane stagnation-point flow, and not that at the leading edge of turbine blades.

From this discussion, it can be seen that the present formulation for the stagnation-point flow problem including surface curvature and external $\Omega$ effects indeed approaches the two limiting cases of $K=0, \Omega \neq 0$ and $K=0$, $\Omega=0$ correctly. Therefore, the solutions obtained are exact and are valid for all values of $R e, k \delta$, and $\Omega$. In addition, the solutions correctly account for the boundary-layer displacement effect at the stagnation point. 
Although one additional initial condition each is required for the integration of (40) and (41), these two equations are not convenient to solve numerically because they involve the integrals of $f$ and $g$. As a result, (35) and (36) are solved, and (44) and (45) are used to estimate the initial values for $f^{\prime \prime}(0)$ and $\theta^{\prime}(0)$ once the initial guesses on $f^{\prime \prime}(0)$ and $g^{\prime}(0)$ are known. The integrals in (44) and (45) are evaluated from the previous iterations for $f$ and $g$. A fourth-order Runge-Kutta technique is used to start the integration of (35) - (37) using the approximate results given in [4] [5] as initial guesses for $f^{\prime \prime}(0), g^{\prime}(0)$, and $\theta^{\prime}(0)$. Subsequent integration of the equations is performed by a predictor-corrector method that has accuracy in both predictor and corrector of $O\left(\Delta \zeta^{5}\right)$. Details of this method are outlined in [25]. The complete integration of equations (35)-(37) is iterated with different initial conditions until the outer boundary conditions (39a)-(39e) are satisfied. The outer boundary conditions are considered satisfied when numerical values at the last two consecutive outer steps agree to within $2 \times 10^{-5}$. When this condition is reached, convergent solutions for $f, g$, and $\theta$ are obtained.

\section{Discussion of Results}

Equations (35)-(37) with boundary conditions (38) and (39) are solved for thirteen different values of $K$; namely, $K=0, \pm 0.015, \pm 0.03, \pm 0.05, \pm 0.1, \pm 0.2, \pm 0.3$, at a $\operatorname{Pr}=0.7$. The integration of $f, g$, and $\theta$ are carried out to $\zeta=10$ for all values of $K$ except $K=-0.1,-0.2$ and -0.3 , and the outer boundary conditions are applied there instead of at $\zeta \rightarrow \infty$. For large negative values of $K$, integrations are carried out to $\zeta=12$; this choice of $\zeta$ is satisfactory because $f, g$, and $\theta$ approach their outer boundary conditions very rapidly even for large values of $|K|$. For all the cases considered, it is found that $f, g$, and $\theta$ approach their free stream value around $\zeta=8$. Hence, the choice of $\zeta=10$ or 12 for all integrations is more than adequate.

Local heat transfer and skin friction can be evaluated from

$$
\begin{gathered}
\dot{q}=\kappa\left(\frac{\partial T}{\partial y}\right)_{y=0}, \\
\tau_{w}=\mu\left(\frac{\partial T}{\partial y}\right)_{y=0} .
\end{gathered}
$$

With the help of (23), (25) and equations (32)-(34), (46) and (47) can be reduced to

$$
\begin{gathered}
\dot{q}=\kappa\left(T_{\infty}-T_{w}\right) \sqrt{\frac{a}{v}} \theta^{\prime}(0), \\
\tau_{w}=\rho a \sqrt{a v} \times f^{\prime \prime}(0)-\mu \Omega g^{\prime}(0) .
\end{gathered}
$$

It can be seen from (49) that the effect of external $\Omega$ is to shift the zero shear point away from the stagnation point, which is located at $x=0$. The shear stress that is responsible for this shift is designated $\left(\tau_{w}\right)_{v}$ and is given by, 


$$
\left(\tau_{w}\right)_{v}=-\mu \Omega g^{\prime}(0) \text {. }
$$

The calculated value is $\left(\tau_{w}\right)_{v}=-1.4065 \mu \Omega$ with displacement effect on $g$ included, and is $\left(\tau_{w}\right)_{v}=-0.6079 \mu \Omega$ when displacement effect is neglected. These results show that $\left(\tau_{w}\right)_{v}$ is not affected by $K$ but depends on $\Omega$ and the displacement. They validate the approximate analysis results of [4] [5], and show that, indeed, the effect of curvature on $g^{\prime}(0)$ is negligible.

If the origin of the coordinate axes is located at the zero-shear point and the new $X$ coordinate is denoted by $\bar{X}$, then (49) can be rewritten as

$$
\bar{\tau}=\rho a \sqrt{a v} \bar{x} f^{\prime \prime}(0) \text {. }
$$

Denoting the local heat transfer and skin friction of the $K=0$ case by a subscript " $O$ ", the following relations for the ratio of local heat transfer and skin friction with and without curvature effect included are obtained

$$
\begin{aligned}
& \frac{\dot{q}}{\dot{q}_{o}}=\frac{\theta^{\prime}(0)}{\theta_{o}^{\prime}(0)}, \\
& \frac{\bar{\tau}}{\bar{\tau}_{o}}=\frac{f^{\prime \prime}(0)}{f_{o}^{\prime \prime}(0)} .
\end{aligned}
$$

These results are shown in Figure 2 and Figure 3, respectively.

In the approximate analysis of [4] [5], the inviscid surface velocity in the immediate vicinity of the stagnation point was expanded in the form

$$
u(x, 0)=u_{11} x+u_{12} x^{2}+O\left(x^{3}\right)
$$

where $u_{11}$ has the dimension of (time $)^{-1}$ and $u_{12}$ has the dimension of $(\text { length })^{-1}$ (time $)^{-1}$. Using this expansion, the approximate analysis gives rise to the following expressions for the local heat transfer and skin friction ratios; namely,

$$
\begin{gathered}
\frac{\dot{q}}{\dot{q}_{o}}=1-0.258356 K+1.810687 \frac{v^{1 / 2} \Omega u_{12}}{u_{11}^{5 / 2}}, \\
\frac{\bar{\tau}}{\bar{\tau}_{o}}=1-1.552226 K+4.743353 \frac{v^{1 / 2} \Omega u_{12}}{u_{11}^{5 / 2}} .
\end{gathered}
$$

On first examination, it seems that the approximate results of [4] [5] are quite reasonable because one would indeed expect the effect of external $\Omega$ to be felt by the local heat flux and the wall shear. The approximate analysis [4] [5] showed that, in addition to shifting the zero-shear point away from the stagnation point, external $\Omega$ also influences local heat transfer and skin friction. However, on closer examination, it could be seen that the inviscid solution (18)-(20) deduced from the present analysis gives an inviscid surface velocity of $u(x, 0)=a x$ only. This implies that the last term in (55) and (56) should be identically zero; i.e., $u_{12} \equiv 0$. In other words, assuming (54) for $u(x, 0)$, as suggested in [4] [5], is incorrect; at least in the immediate vicinity of the stagnation point. Setting $u_{12}=0$ reduce the equations for local heat flux (55) and local shear stress (56) to: 


$$
\begin{gathered}
\frac{\dot{q}}{\dot{q}_{o}}=1-0.258356 K, \\
\frac{\bar{\tau}}{\bar{\tau}_{o}}=1-1.552226 \mathrm{~K} .
\end{gathered}
$$

These results are shown in Figure 2 and Figure 3 for comparison.

It can be seen in Figure 2 that the exact wall heat flux decrease with $K$ for $K>$ 0 is faster than that predicted by the approximate analysis [4] [5], and the opposite is true for $K<0$. But as shown in Figure 3, there is no discernible difference between the exact result for wall shear stress and that obtained from the approximate analysis [4] [5]. However, on close examination, a consistent difference does exist, especially for large $K$. This is evident from the results tabulated in Table 1 where the values of $f^{\prime \prime}(0)$ and $\theta^{\prime}(0)$ are reported to the fourth decimal point. The approximate results [4] [5], which are denoted by a subscript VD, are also listed in Table 1 for comparison. It can be seen that the approximate results are correct for values of $K$ up to $|K|=0.015$. Thereafter, they deviate from the exact solutions. The variations are small for small values of $|K|$,

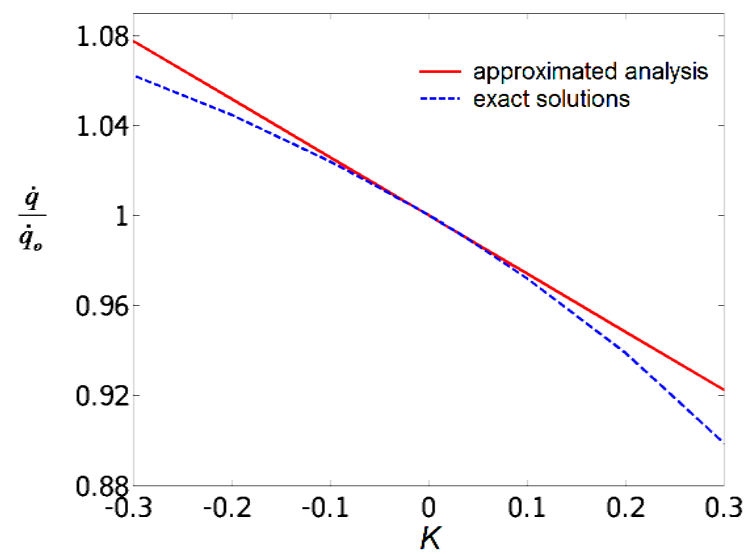

Figure 2. Wall heat flux, $\dot{q} / \dot{q}_{0}$, at different values of $K$ (red solid line-approximate analysis [4] [5]; blue dashed line-exact solutions).

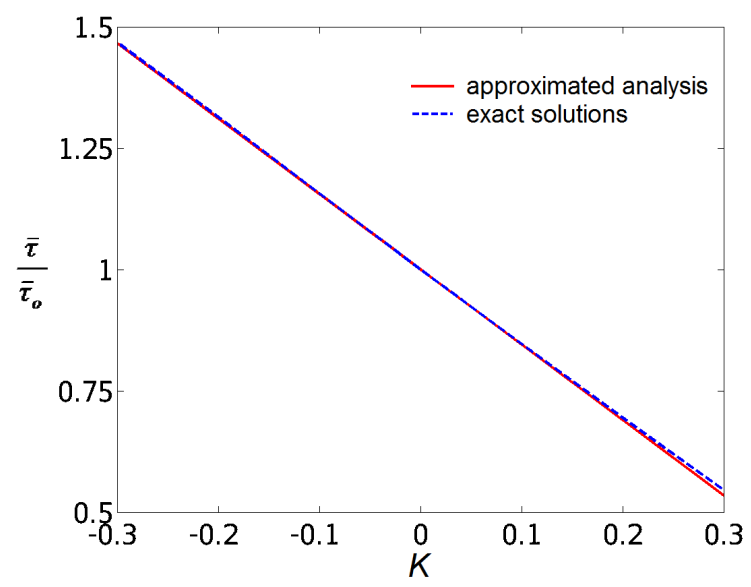

Figure 3. Wall shear stress, $\bar{\tau} / \bar{\tau}_{o}$, at different values of $K$ (red solid line-approximate analysis [4] [5]; blue dashed line-exact solutions). 
Table 1. A comparison of $f^{\prime \prime}(0)$ and $\theta^{\prime}(0)$ with approximate results deduced from [4] [5] for different values of $K$.

\begin{tabular}{|c|c|c|c|c|c|c|c|c|c|c|c|c|c|}
\hline & \multicolumn{13}{|c|}{$K$} \\
\hline & -0.3 & -0.2 & -0.1 & -0.05 & -0.03 & -0.15 & 0 & 0.15 & 0.03 & 0.05 & 0.1 & 0.2 & 0.3 \\
\hline$f^{\prime \prime}(0)$ & 1.8092 & 1.6188 & 1.4250 & 1.3286 & 1.2901 & 1.26131 & 1.2326 & 1.2039 & 1.1753 & 1.1372 & 1.0426 & 0.8553 & 0.6717 \\
\hline$f_{V D}^{\prime \prime}(0)$ & 1.8066 & 1.6152 & 1.4239 & 1.3283 & 1.2900 & 1.26131 & 1.2326 & 1.2039 & 1.1752 & 1.1369 & 1.0413 & 0.8499 & 0.6586 \\
\hline$\theta^{\prime}(0)$ & 0.5268 & 0.5781 & 0.5078 & 0.5021 & 0.4996 & 0.49780 & 0.4959 & 0.4959 & 0.4920 & 0.4892 & 0.4820 & 0.4656 & 0.4457 \\
\hline$\theta_{V D}^{\prime}(0)$ & 0.5343 & 0.5215 & 0.5087 & 70.5023 & 0.4997 & 0.49780 & 0.4959 & 0.4959 & 0.4920 & 0.4895 & 0.4895 & 0.4702 & 0.4574 \\
\hline
\end{tabular}

but increase as $|K|$ increases. These small differences are significant because in the course of numerically integrating (35) and (37), it is found that, if the approximate values are used for $f^{\prime \prime}(0)$ and $\theta^{\prime}(0)$, the numerical results fail to approach the boundary conditions at $\zeta=10$ or 12 correctly and still satisfy the accuracy criteria imposed on $f^{\prime \prime}(\zeta), f^{\prime \prime \prime}(\zeta)$, and $\theta(\zeta)$. The situation gets worse when $|K|$ increases and at $|K| \geq 0.1$ the numerical computations fail to converge to a meaningful solution. Consequently, it can be concluded that the approximate analysis of [4] [5] gives the correct slope for $\bar{\tau} / \bar{\tau}_{o}$ and $\dot{q} / \dot{q}_{o}$ at $K$ $=0$ only. This was pointed out in [21] where the authors concluded that there is actually no justification for attaching any significance to the curvature of the curves for $\bar{\tau} / \bar{\tau}_{o}$ and $\dot{q} / \dot{q}_{o}$ when they are deduced from any one of the known approximate methods [19] [20] [21].

The higher wall shear given by the exact analysis is a direct consequence of the more favorable pressure gradient seen by the flow for a given $K$. From (2) it can be deduced that the pressure gradient at the wall along the flow direction is given by

$$
\rho^{-1}\left(\frac{\partial p}{\partial x}\right)_{y=0}=v\left(\frac{\partial^{2} u}{\partial y^{2}}\right)_{y=0}+v k\left(\frac{\partial u}{\partial y}\right)_{y=0} .
$$

With the help of (23) and equations (31) - (33), it can be shown that (59) reduces to

$$
\rho^{-1}\left(\frac{\partial p}{\partial x}\right)_{y=0}=a^{2} x\left[f^{\prime \prime \prime}(0)-2 K f^{\prime \prime}(0)\right]-\nu \Omega\left[g^{\prime \prime}(0)-2 K g^{\prime}(0)\right] .
$$

Again, the effect of external $\Omega$ is to shift the zero pressure gradient point away from the stagnation point. Consequently, (60) can be written as

$$
\frac{(\partial p / \partial x)_{y=0}}{\rho a^{2} \bar{x}}=f^{\prime \prime \prime}(0)-2 K f^{\prime \prime}(0),
$$

while the corresponding result deduced from the approximate analysis [4] [5] is

$$
\left[\frac{(\partial p / \partial x)_{y=0}}{\rho a^{2} \bar{x}}\right]_{V D}=-1+1.880488 K .
$$

These results are plotted in Figure 4. They show that the decrease in favorable pressure gradient with $K$, for $K>0$, is faster for the exact solution than for the 


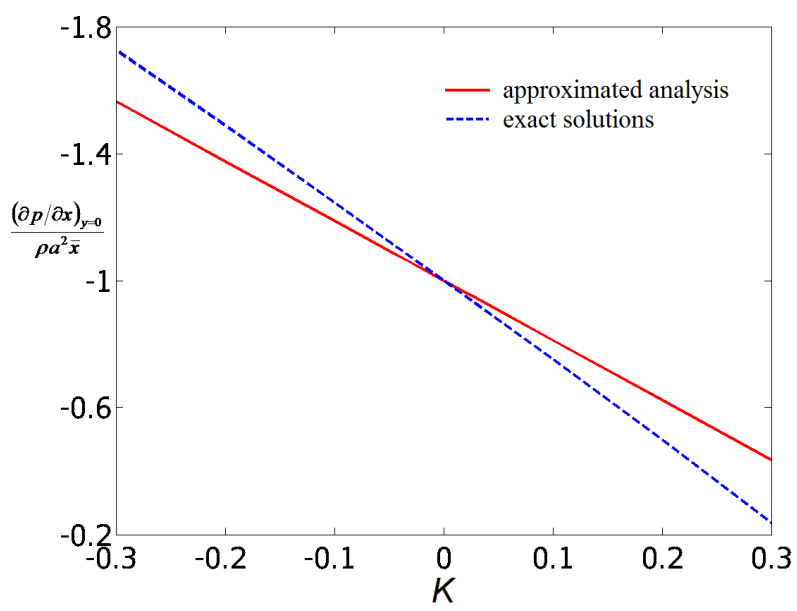

Figure 4. Wall pressure gradient ratio, $(\partial p / \partial x)_{y=0} / \rho a^{2} \bar{x}$, at different values of $K$ (red solid line-approximate analysis [4] [5]; blue dashed line-exact solutions).

approximate analysis. The opposite is true for $K<0$.

Some sample plots of $f, f^{\prime}, f^{\prime \prime}, g, g^{\prime}$, and $q$ for three different values of $K$ are shown in Figures 5-10, respectively. They clearly demonstrate the effect of curvature on these profiles. Therefore, if the plane stagnation-point-flow solution is used as initial conditions for subsequent heat transfer calculations around turbine blades, the error incurred could be substantial, depending on the nose curvature and the external $\Omega$. The latter effect would shift the zero shear point and the zero pressure gradient point away from the stagnation point, and this could have an impact on transition to turbulence and local separation. However, curvature would affect the wall shear, the wall heat flux, the pressure distribution around the leading edge and, more importantly, the boundary layer thickness in the vicinity of the stagnation point (Figure 6). This means that if the $K=0$ solution is used as initial conditions, a wrong estimate of the velocity profile and momentum and displacement thicknesses would have been used for subsequent heat transfer calculation around turbine blades. The seriousness of this error is best illustrated by examining the variation of the displacement thickness ratio, $\delta^{*} / \delta_{o}^{*}$, with $K$ for the case $\Omega=0$ (Figure 11 ), which clearly underlines the importance of including surface curvature effect in the calculation of leading edge heat transfer.

Finally, consider the determination of " $a$ " which is as yet undefined. To accomplish this, consider the flow along the stagnation streamline toward a circular cylinder in the absence of external vorticity. In the vicinity of the stagnation point, the velocity along the stagnation streamline as given by (i) the plane stagnation-point flow inviscid solution, (ii) the present inviscid solution, and (iii) the potential solution around a cylinder, can be written as

(i) $\frac{V_{o}^{p}}{V_{\infty}}=-\frac{a y}{V_{\infty}} \quad$ (plane wall)

(ii) $\frac{V_{o}^{c}}{V_{\infty}}=-\frac{a R^{2} \ln (1+y / R)}{V_{\infty}(R+y)}$ (curved wall) 


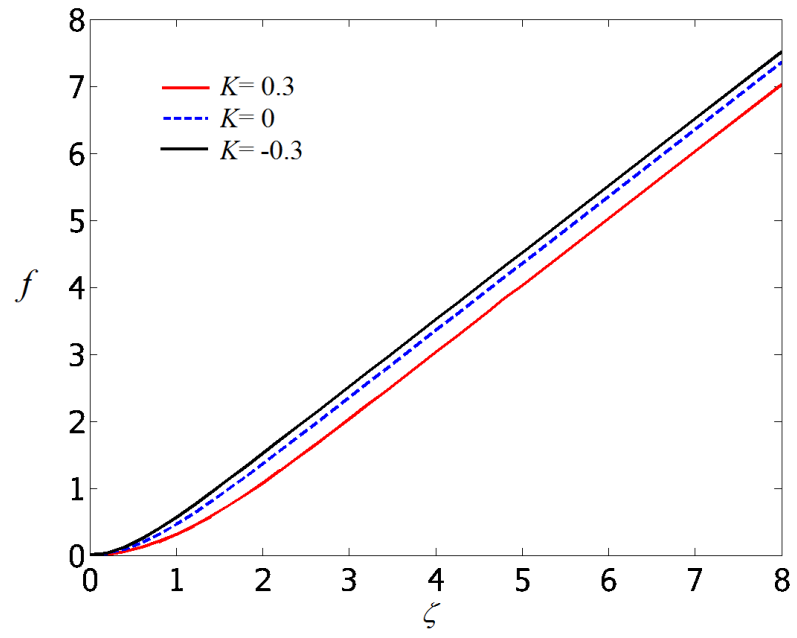

Figure 5. Effects of surface curvature on $f$ (red solid line $-K=0.3$; blue dashed line $-K=$ 0 ; black solid line $-K=-0.3$ ).

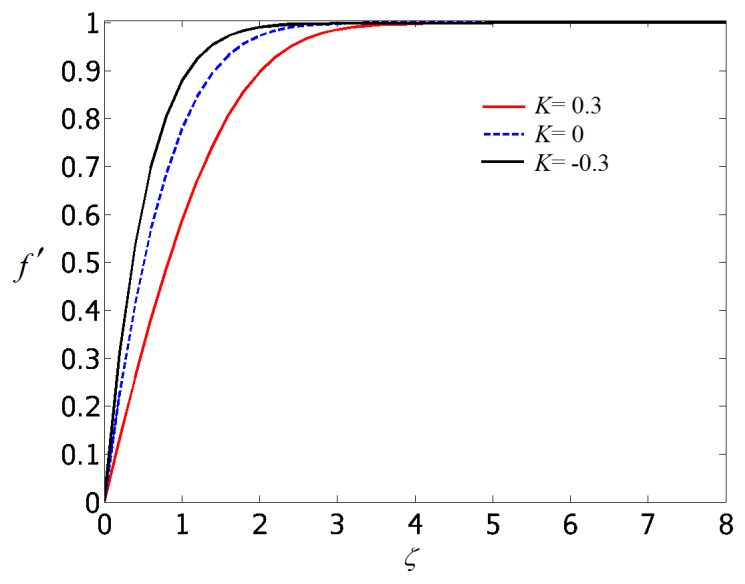

Figure 6. Effects of surface curvature on $f^{\prime}$ (red solid line $-K=0.3$; blue dashed line $-K=0$; black solid line $-K=-0.3$ ).

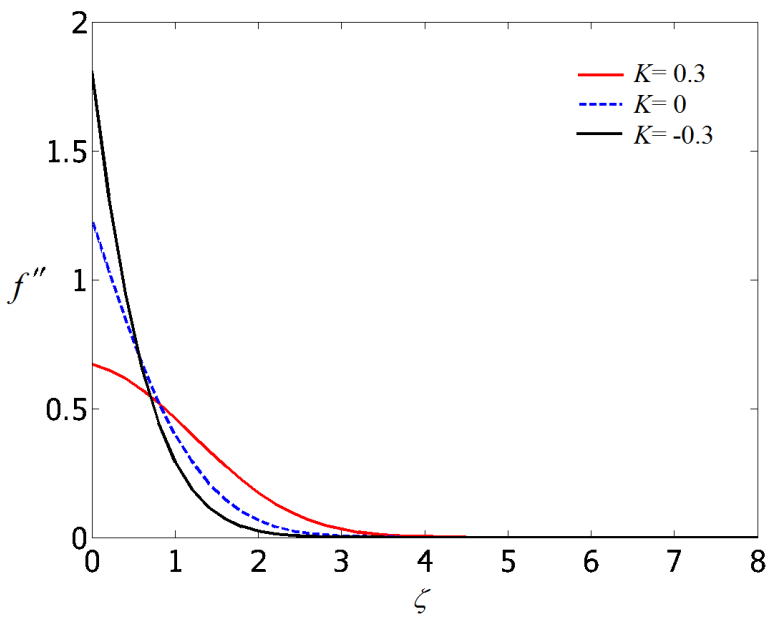

Figure 7. Effects of surface curvature on $f^{\prime \prime}$ (red solid line $-K=0.3$; blue dashed line $-K=0$; black solid line $-K=-0.3$ ). 


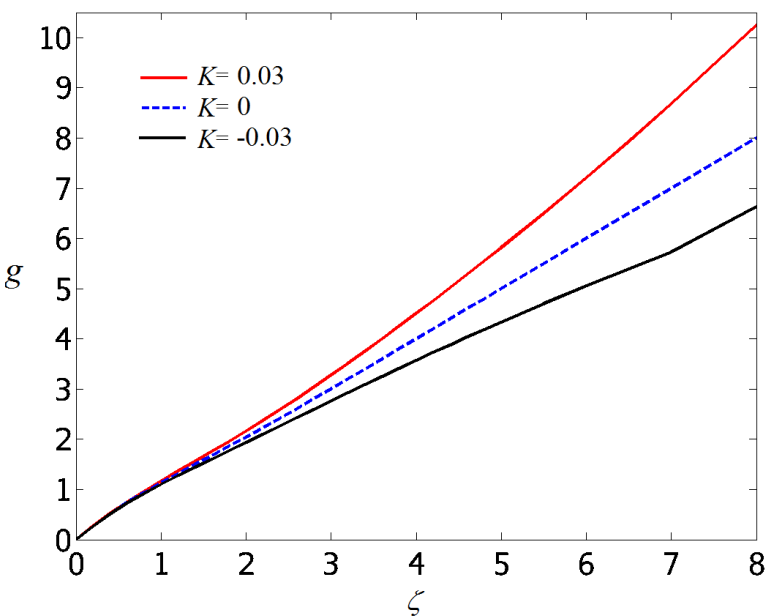

(a)

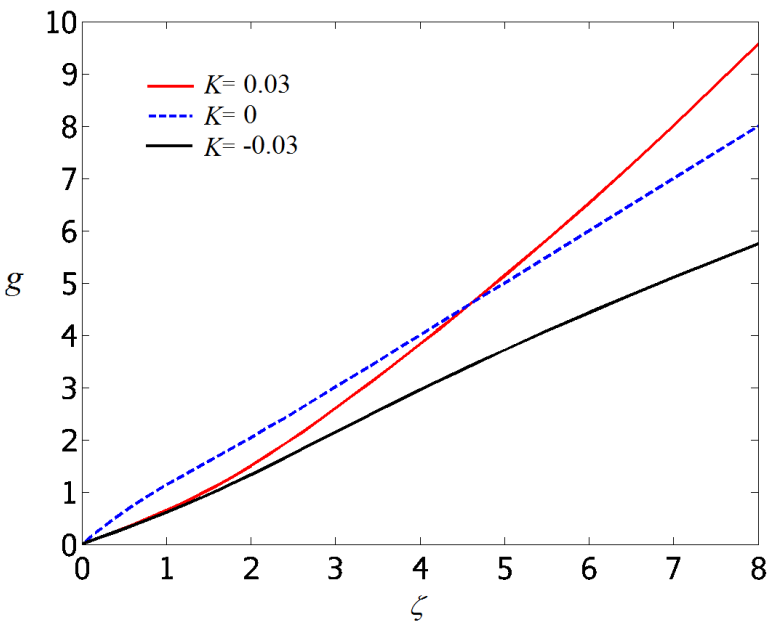

(b)

Figure 8. (a) Effects of surface curvature on $g$ with displacement effect (red solid line $-K$ $=0.03$; blue dashed line $-K=0$; black solid line $-K=-0.03$ ); Effects of surface curvature on $g$ without displacement effect (red solid line $-K=0.03$; blue dashed line $-K=0$; black solid line $-K=-0.03)$.

(iii) $\frac{V_{o}}{V_{\infty}}=-\frac{y(y+2 R)}{(R+y)^{2}}$ (cylinder)

where $V_{\infty}$ is the velocity far upstream and $V_{o}^{p}, V_{o}^{c}, V_{o}$ are the velocities along the stagnation streamlines for the three cases (i), (ii), (iii) considered, respectively. For very small $y$, the above relations reduce to

$$
\frac{V_{o}^{p}}{V_{\infty}}=\frac{V_{o}^{c}}{V_{\infty}}=\frac{V_{o}}{V_{\infty}}=-\frac{2 y}{R}=-\frac{a y}{V_{\infty}},
$$

thus giving,

$$
a=\frac{2 V_{\infty}}{R} .
$$

Therefore, " $a$ " can be determined from knowledge of the approach flow and the nose radius. 


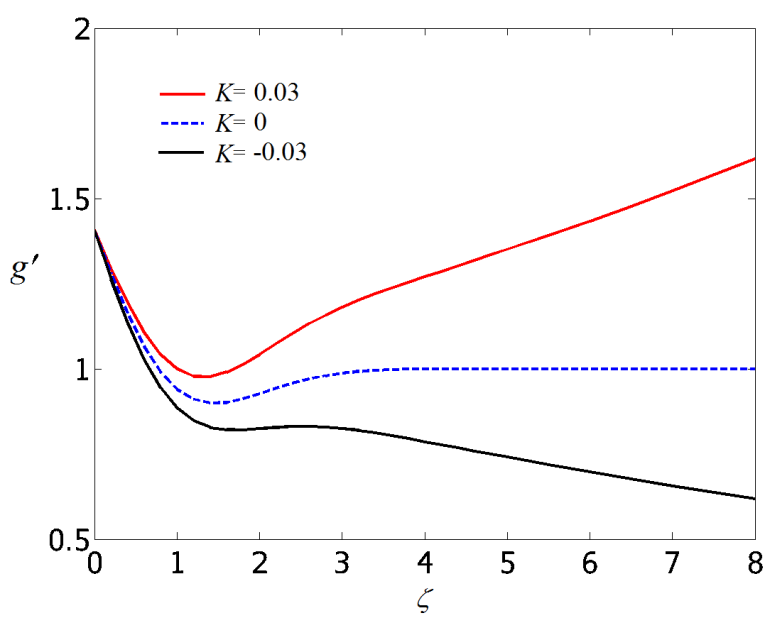

(a)

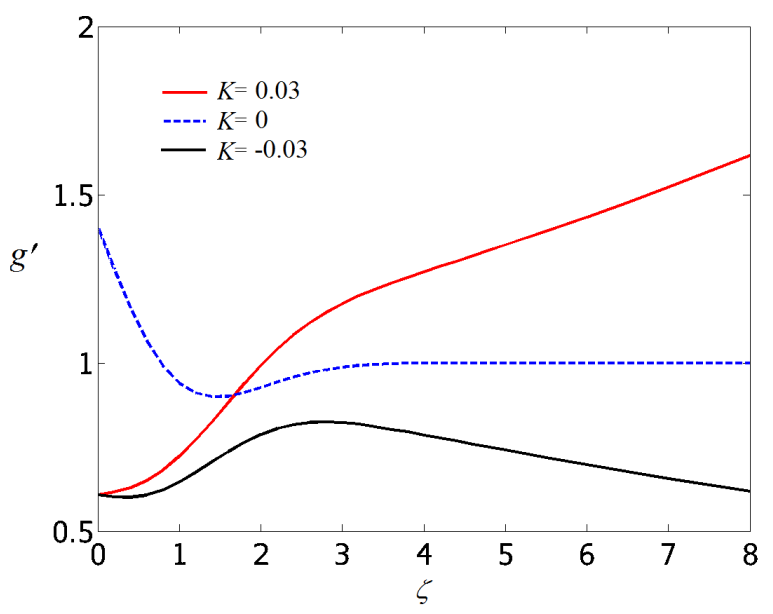

(b)

Figure 9. (a) Effects of surface curvature on $g^{\prime}$ with displacement effect (red solid line $-K=0.03$; blue dashed line $-K=0$; black solid line $-K=-0.03$ ); (b) Effects of surface curvature on $g^{\prime}$ without displacement effect (red solid line $-K=0.03$; blue dashed line $-K=0$; black solid line $-K=-0.03$ ).

At this point, it can be concluded that the thermal stagnation-point flow problem at the leading edge of axial flow turbine blades has been correctly and completely solved. This leads to exact solutions for the governing Navier-Stokes equations under the assumption of steady flow, small nose radius, constant external $\Omega$, and any $R e$.

\section{Extention to Centrifugal Impeller Blades}

The present analysis is formulated for a stationary curved surface with a uniform flow having a constant $\Omega$ approaching the surface. Therefore, it is directly applicable to leading edge problems in axial flow turbines where Coriolis force effect are absent in the viscous flow and heat transfer around turbine blades. However, this is not true for a radial flow machine, and it would seem that the present analysis could not be applied to study the leading edge problem of cen- 


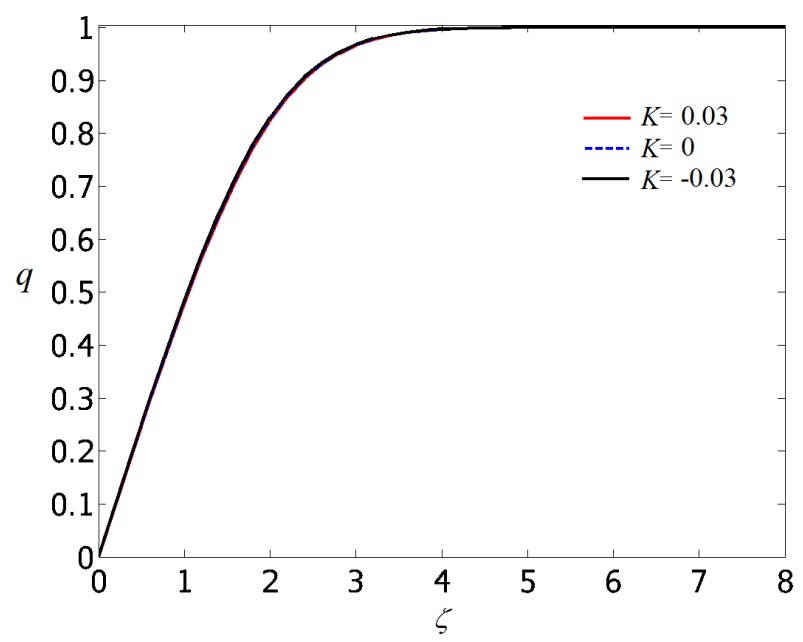

Figure 10. Effects of surface curvature on $q$ (red solid line $-K=0.03$; blue dashed line $-K=0$; black solid line $-K=-0.03$ ).

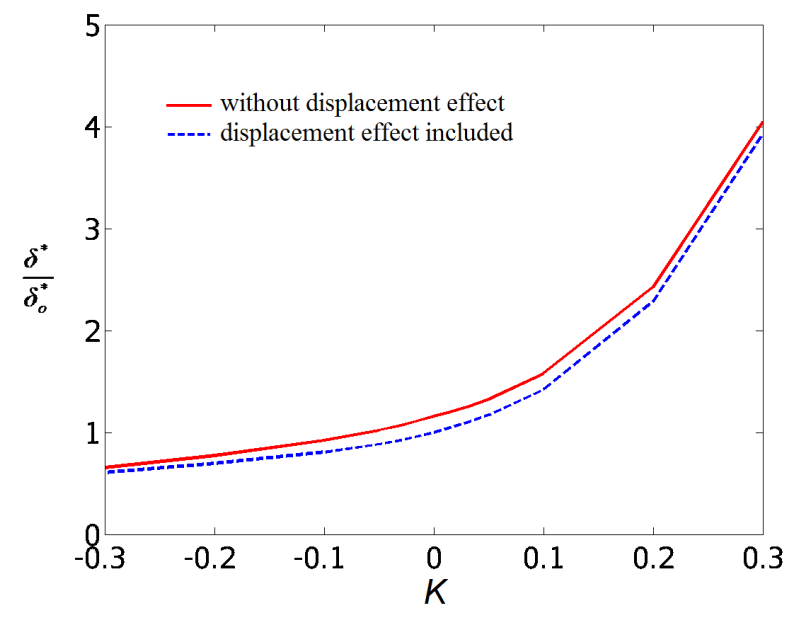

Figure 11. Effects of surface curvature on the displacement thickness ratio, $\delta^{*} / \delta_{o}^{*}$, for the case $\Omega=0$ (red solid line-without displacement effect; blue dashed line-displacement effect included).

trifugal impeller blades. Fortunately, there are similarities between a uniform shear flow toward a stationary surface and a uniform flow toward a rotating surface that would allow the use of the present results for the study of flows toward rotating curved surfaces.

To appreciate this, consider the steady thermal stagnation-point flow with a uniform velocity approaching a two-dimensional curved surface rotating at a constant speed of $\Omega / 2$. The equations governing the two-dimensional flow can be written with respect to a coordinate system attached to the rotating surface (Figure 12) and obeying the right-hand rule. If $u$ and $v$ are again used to denote the relative velocities along $x$ and $y$ direction, respectively, then the steady state equations can be written as

$$
u_{x}+(h v)_{y}=0
$$




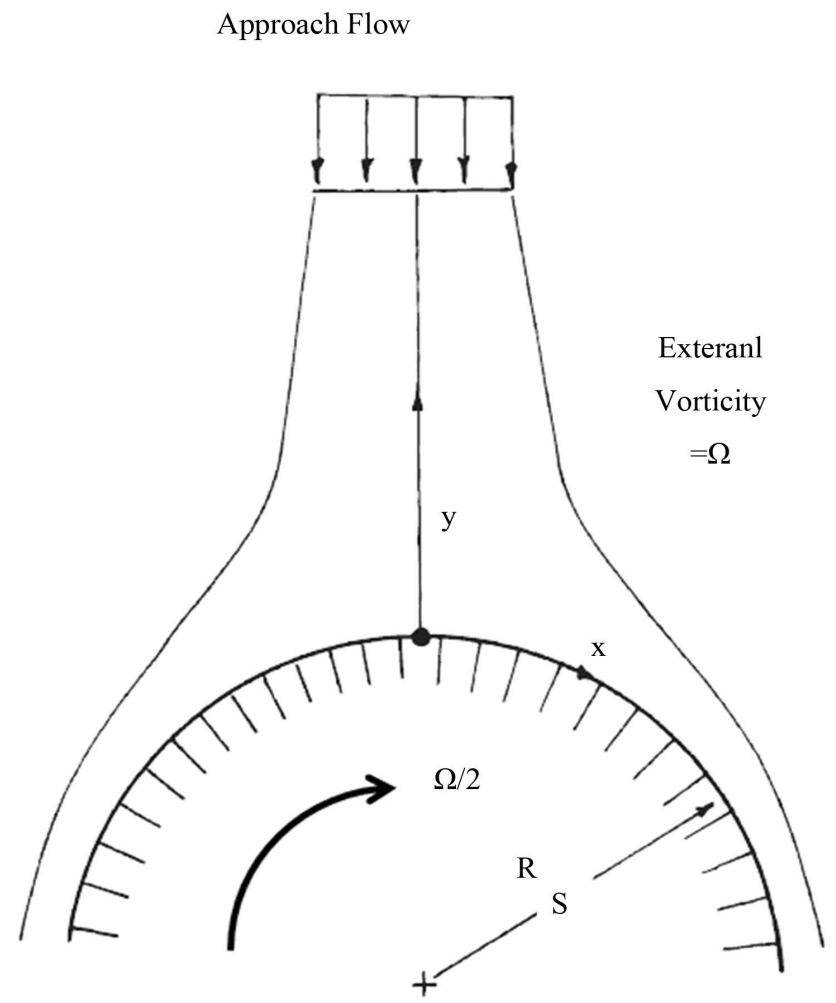

Figure 12. Curvilinear co-ordinate system for a rotating surface with constant speed of $\Omega / 2$.

$$
\begin{gathered}
u u_{x}+v(h u)_{y}-h \Omega v=-\rho^{-1} p_{x}^{*}+v\left[h^{-1} u_{x x}+h u_{y y}+k u_{y}-k^{2} h^{-1} u+2 k h^{-1} v_{x}\right], \\
u v_{x}+h v v_{y}-k u^{2}+h \Omega u=-\rho^{-1} h p_{y}^{*}+v\left[h^{-1} v_{x x}+h v_{y y}+k v_{y}-k^{2} h^{-1} v-2 k h^{-1} u_{x}\right] \\
u T_{x}+h v T_{y}=\alpha\left[h^{-1} T_{x x}+h T v_{y y}+k T_{y}\right] \\
\text { where } p^{*}=p-\frac{1}{2} \rho r^{2}\left(\frac{\Omega}{2}\right)^{2},
\end{gathered}
$$

is the reduced pressure and $\mathrm{r}$ is the radial distance from the axis of rotation. As before, $k$ is taken to be constant. Since the flow is two-dimensional, there is only one component of vorticity and it is normal to the plane of flow. This component is given by

$$
h^{-1} v_{x}-h^{-1}(h u)_{y}=\Omega,
$$

in the inviscid flow region.

With the problem thus formulated, it can be easily seen that the inviscid flow is again governed by the Poisson Equation (5) with boundary conditions given by (8) and (9). Therefore, the inviscid velocity field is given by (18) and (19) and the pressure field is obtained by integrating (66) and (67) with the kinematic viscosity $v \equiv 0$. The result is

$$
p_{o}^{*}-p^{*}=\frac{1}{2} \rho\left[\frac{a^{2} x^{2}}{h^{2}}+\frac{a \Omega x}{k} \frac{4 h^{2} \ln h-h^{2}+1}{h^{2}}-\frac{\Omega^{2}}{4 k^{2}} \frac{h^{4}+1}{2 h^{2}}+\frac{a^{2}(\ln h)^{2}}{k^{2} h^{2}}\right] .
$$


By cross-differentiating Equations (66) and (67) to eliminate $p^{*}$, it can be shown that the resulting vorticity equation is again given by (21) because the Coriolis force terms are zero as a result of the continuity equation (65). The boundary conditions are the same as (22). In view of this, the velocity field around the stagnation point of a rotating curved surface is the same as that around the stagnation point of a stationary curved surface with an approach flow having a constant $\Omega$ equal to twice the rotational speed. Since the temperature field as defined by (68) only depends on the velocity field, the resulting solution of (68) would also be the same as that obtained before, provided the thermal boundary conditions remain the same. The pressure field will be different and is given by the integral of (66) and (67) once the velocity field is known.

From the above discussion, it can be seen that the present exact analysis can also be extended to study the steady heat transfer and viscous flow around the stagnation point at the leading edge of centrifugal impeller blades.

\section{Conclusions}

The problem of a steady thermal stagnation-point flow at the leading edge of an axial flow turbine under the influence of a constant external $\Omega$ has been analyzed. It is shown that exact solutions to the steady governing NavierStokes equations can be obtained if the nose radius is constant. Heat transfer and skin friction results obtained for the curvature parameter $K$ ranging from $-0.3 \leq K \leq 0.3$ show that, within this range, the linear relation between wall shear and $K$ given by the approximate analysis of [4] [5] is essentially correct. However, the decrease of wall heat flux with $K$ for $K>0$ is faster than that predicted in [4] [5], and the opposite is true for $K<0$. Consequently, the exact results show that the approximate analysis is correct only in the determination of the slopes of the variation of wall shear and local heat transfer with $K$ at $K=0$.

External $\Omega$ gives rise to a wall shear stress that is responsible for shifting the zero skin friction point away from the stagnation point. Although the wall shear is a function of external $\Omega$ and displacement, it is independent of curvature. Besides this effect, external $\Omega$ has no other effect on wall shear and local heat transfer. This is contrary to the results given in [4] [5], which revealed that external $\Omega$ also has a second-order effect on wall shear and wall heat flux. This error in the analysis detailed in [4] [5] could be traced to its incorrect proposed expansion for the inviscid surface velocity in the immediate vicinity of the stagnation point. If a correct expansion is proposed, the approximate results are consistent with the exact solutions, at least to the lowest order.

Surface curvature also influences the wall static pressure distribution in the vicinity of the stagnation point. It is found that convex curvature decreases the favorable pressure gradient, but concave curvature increases it. This implies that the flow near the leading edge of turbine blades (whose curvature is convex) will be more susceptible to laminar separation than the corresponding flow toward a plane surface. In addition, surface curvature affects the velocity and temperature profiles and the thickness of the viscous layer. All these underline the impor- 
tance of including surface curvature and external $\Omega$ effect in the heat transfer calculation around turbine blades.

Finally, it is shown that the present analysis can also be applied to study the steady leading edge stagnation-point flow problem in centrifugal impeller blades. With the exception of the pressure field, the solution to the impeller blade problem is identical to that of the axial flow turbine blade.

\section{Acknowledgements}

The first author would like to acknowledge the support given him by the Mechanical Engineering Department, the Hong Kong Polytechnic University, Hong Kong, during his stay as Visiting Chair Professor from September to October of 2016.

\section{References}

[1] van Driest, E.R. (1959) Convective Heat Transfer in Gases. In: Lin, C.C., Ed., High Speed Aerodynamics and Jet Propulsion, Vol. 5, Princeton University Press, Princeton, NJ, 339.

[2] Kline, S.J., Morkovin, M.V., Sovran, G. and Cockrell, D.J. (1968) v.1. Methods, Predictions, Evaluation, and Flow Structure. Proceedings of the Stanford Conference on Computation of Turbulent Boundary Layers, AFOSR-IFP, 1, 18-19.

[3] Coles, D.E. and Hirst, E.A. (1968) v.2.Compiled Data. Proceedings of the Stanford Conference on Computation of Turbulent Boundary Layers, AFOSR-IFP, 2.

[4] Van Dyke, M. (1962) Higher Approximations in Boundary Layer Theory, Part I: General Analysis. Journal of Fluid Mechanics, 14, 161-177. https://doi.org/10.1017/S0022112062001147

[5] Van Dyke, M. (1962) Higher Approximations in Boundary Layer Theory, Part II: Application to Leading Edges. Journal of Fluid Mechanics, 14, 481-495. https://doi.org/10.1017/S0022112062001391

[6] Bradshaw, P. (1973) Effect of Streamline Curvature on Turbulent Flow. AGARDograph 169.

[7] So, R.M.C. and Mellor, G.L. (1973) Experiment on Convex Curvature Effect in Turbulent Boundary Layers. Journal of Fluid Mechanics, 60, 43-62.

https://doi.org/10.1017/S0022112073000030

[8] So, R.M.C. and Mellor, G.L. (1975) Experiment on Turbulent Boundary Layers on a Concave Wall. Aeronautical Quarterly, 26, 25-40. https://doi.org/10.1017/S0001925900007174

[9] Bradshaw, P. (1969) The Analogy between Streamline Curvature and Buoyancy in Turbulent Shear Flow. Journal of Fluid Mechanics, 36, 177-191. https://doi.org/10.1017/S0022112069001583

[10] Rastogi, A.K. and Whitelaw, J.H. (1971) Procedure for Predicting the Influence of Longitudinal Curvature on Boundary Layer Flows. ASME Paper 71-WA/FE-37.

[11] Irwin, H.P.A.H. and Arnot Smith, P. (1975) Prediction of the Effect of Streamline Curvature on Turbulence. Physics of Fluids, 18, 624-630. https://doi.org/10.1063/1.861202

[12] So, R.M.C. and Mellor, G.L. (1978) Turbulent Boundary Layers with Large Streamline Curvature Effect. Zeitschrift für angewandte Mathematik und Physik ZAMP, 
29, 54-74. https://doi.org/10.1007/BF01797303

[13] Johnston, J.P. and Eide, S.A. (1976) Turbulent Boundary Layers on Centrifugal Compressor Blades, Prediction of the Effect of Surface Curvature and Rotation. Journal of Fluids Engineering, 98, 374-381.

[14] So, R.M.C. (1981) Heat Transfer Modeling for Turbulent Shear Flows on Curved Surfaces. Zeitschrift für angewandte Mathematik und Physik ZAMP, 32, 514-532. https://doi.org/10.1007/BF00947018

[15] Koosinlin, M.L., Launder, B.E. and Sharma, B.I. (1974) Prediction of Momentum, Heat and Mass Transfer in Swirling, Turbulent Boundary Layers. Journal of Heat Transfer, 96, 204-209. https://doi.org/10.1115/1.3450165

[16] Hiemenz, K. (1911) Die Grenzschicht an einem in den gleichformigen Flussigkeitsstrom eingetauchten geraden Kreiszylinder. Dingler's Polytechnical Journal, 326, 321.

[17] Howarth, L. (1938) On the Calculation of the Steady Flow in the Boundary Layer near the Surface of a Cylinder in a Stream. Aeronautical Research Council, R. \& M. 1632.

[18] Schlichting, H. (1960) Boundary Layer Theory. 4th Edition, McGraw-Hill Book Company, New York, London, 78.

[19] Massey, B.S. and Clayton, B.R. (1965) Laminar Boundary Layers and Their Separation from Curved Surfaces. Journal of Basic Engineering, 87, 483-493. https://doi.org/10.1115/1.3650580

[20] Schultz-Grunow, F. and Breuer, W. (1965) Laminar Boundary Layers on Cambered Walls. In: Holt, M., Ed., Basic Developments in Fluid Dynamics, Vol. 1, 377-436.

[21] Narasimha, R. and Ojha, S.K. (1967) Effect of Longitudinal Surface Curvature on Boundary Layers. Journal of Fluid Mechanics, 29, 187-199. https://doi.org/10.1017/S0022112067000710

[22] Stuart, J.T. (1959) The Viscous Flow Near a Stagnation Point When the External Flow Has Uniform Vorticity. Journal of the Aerospace Sciences, 26, 124-125. https://doi.org/10.2514/8.7963

[23] van Dyke, M. (1969) Higher-Order Boundary Layer Theory. Annual Review of Fluid Mechanics, 1, 265-292. https://doi.org/10.1146/annurev.fl.01.010169.001405

[24] Goldstein, S. (1965) Modern Developments in Fluid Dynamics. Vo. 1, Dover Publications, Inc., New York, 119.

[25] Ralston, A. and Wilf, H.S. (1960) Mathematical Methods for Digital Computers. John Wiley Sons, Inc., New York, London, 93. 


$\begin{array}{ll}\kappa & \text { Thermal conductivity of fluid } \\ \mu & \text { Viscosity of fluid } \\ v & \text { Fluid kinematic viscosity } \\ \rho & \text { Fluid density } \\ \tau_{W} & \text { Wall shear stress } \\ \bar{\tau} & \text { Modified wall shear stress } \\ \bar{\tau}_{0} & \text { Modified wall shear stress for the } k=0 \text { case } \\ \Psi & \text { Stream function for inviscid flow } \\ \Omega & \text { External vorticity } \\ \zeta=\ln (1+K \eta) / K \text { Transformed similarity variable }\end{array}$

Arbitrary constant defined in Equation (13)

Arbitrary constant defined in Equation (14)

Arbitrary constant defined in Equation (16)

Specific heat of fluid at constant pressure

Similar velocity function defined in Equation (23)

$$
\text { Metric coefficient }
$$

\section{Surface curvature}

Normalized surface curvature

Characteristic length

Static pressure

Static pressure at the stagnation point

Prandtl number

Wall heat flux

Wall heat flux for the $k=0$ case

Reynolds number

Fluid temperature

Wall temperature

Fluid temperature far away from the wall

Velocity along $\mathrm{x}$-direction

Characteristic velocity

Velocity along y-direction

Thermal diffusivity of fluid

Thickness of viscous layer

Displacement thickness for the $\Omega=0$ case

Similarity variable The

Integration constants defined in Equation (40) and Equation (41)

Transformed similar velocity function defined in Equation (32)

Similar velocity function defined in Equations (23) and (24)

Transformed similar velocity function defined in Equation (33)

Coordinates measured along the wall and normal to the wall, re-

Nondimensional temperature function defined in Equation (25)

Transformed nondimensional temperature function defined in 